\title{
Centralisation et décentralisation aux Pays-Bas à 1'époque contemporaine
}

\author{
P.J. VERDAM
}

En 1795, l'entrée des armées francaises dans les Pays-Bas du Nord marqua la fin de la République des Sept Provinces-Unies. Cela mit aussi un terme à la souveraineté des diverses provinces et à l'assujettissement des pays situés au sud des grands fleuves (les pays de la Généralité). Un état unitaire se constitua à la place. La vieille question de savoir quel était le pouvoir des Etats Généraux à cóté de la souveraineté des provinces fit alors place à un nouveau dilemme: quelle était la position des provinces à 1'intérieur du nouvel état?

Les idees centralistes caractérisent de nombreuses révolutions et ce fut également le cas ici. Les choses débutèrent evidemment bien. Il devait se constituer un état central et tous ceux qui n'avaient pas fui ou n'avaient pas été exilés étaient d'accord sur ce point. Dans 1'intervalle, la discussion s'engagea entre ceux qui étaient restés. L'un des point majeurs de la discussion est contenu dans le nom même des deux partis que s'affrontaient: Fédéralistes et Unitaristes. J'estime inutile d'exposer ici les discussions qui se déroulèrent alors. Les noms des partis en caractérisent le contenu; le dix-huitième siècle fut le siècle de l'éloquence et les reformateurs sont aussi des écrivains prolixes. La Relation au jour le jour (Dagverhaal) est interminable. Le premier projet de régime politique est rejeté par 1'Assemblée Nationale. Un second projet bascule dans le referendum. On devine l'issue inévitable: un coup d'état. Et en effet, les Unitaristes expulsent cavalièrement les Fédéralistes de 1'Assemblee Nationale. C'est ainsi que la première constitution peut être promulguée au cours de cette même année 1798.

Le contenu de cette constitution unitariste va de soi. Même le mot de province n'y figure plus. Il y a dorénavant huit départements et leurs limites sont le plus possible distinctes de celles des anciennes provinces. Ces départements ne jouaient aucun rôle dans la composition des organes nationaux; des districts aux limites à nouveau différentes furent créés dans ce but. Les corps administratifs départementaux conservaient leur caractère collégial mais étaient présidés par un commissaire dont le rôle consistait a assurer la plus stricte observation des lois et des décrets. Il ne subsistait plus rien du rôle autonome propre des provinces. Les départements n'obtinrent pas non plus de participation dans 1'exercice de l'admi- 


\section{P.J. VERDAM}

nistration nationale; ils n'étaient que des exécutants du pouvoir central. Ils devaient obtenir leurs moyens financiers du gouvernement central. Tous les citoyens jouissant du droit de vote devaient signer une déclaration dans laquelle ils affirmaient non seulement leur aversion pour la Maison d'Orange mais aussi pour le federalisme. Tel fut donc le régime ultra-centraliste de 1798. Au cours des années suivantes, l'influence française devint prépondérante. Une unique fois, cela signifie que 1'accent est mis sur le traitement bienveillant dont le premier consul français fait preuve à l'égard des pays voisins pour l'amour de la paix et c'est ainsi que la constitution de 1801 conféra aux départements les anciennes limites des provinces et que les départements obtinrent un assez grand nombre de compétences. Par l'instauration du Royaume de Hollande, en 1806, sous Louis Bonaparte, frère de Napoléon, la centralisation fut rétablie dans les Pays-Bas septentrionaux; cette centralisation fut poursuivie et accentuée par l'annexion et le rattachement à la France en 1810.

Après la chute de Napoléon, les Pays-Bas devinrent un état unitaire sous Guillaume d'Orange, fils du dernier stadhouder décédé entretemps. Les anciennes discussions se ranimèrent spontanément lors de la rédaction de la constitution. Une question resta cependant hors des débats: les Pays-Bas du Nord constitueraient un état unitaire dont Guillaume d'Orange serait le prince souverain. Les discussions aboutirent en définitive à un compromis de tendance fortement centraliste. Cette constitution de 1814 a posé les bases d'une situation qui se prolonge encore jusqu'è ce jour. Les principaux points en sont les suivants:

1. Les limites des provinces sont pratiquement identiques aux anciennes; leurs organes administratifs retrouvent le nom d'Etats et ils sont élus par les habitants. Une position privilégiée est accordee aux nobles, mais cela ne fut que de courte durée.

2. Le pouvoir des Etats provinciaux est en fait tres limité. La limitation à l'application des lois est cependant jugée trop restreinte. Le reglement des affaires intérieures de la province appartiendra également à leurs attributions.

3. Les provinces n'obtiennent pas de moyens financiers propres. Elles demandent au roi de faire figurer les moyens nécessaires sur le budget de l'Etat.

4. Les Etats provinciaux obtiennent le pouvoir d'élire des membres du parlement (ce sont eux qui élisent encore actuellement les membres de la Première Chambre).

5. Le college des Etats députés forme la commission permanente de la province. 6. Un commissaire représentant le prince souverain veillera, dans chaque province, à l'exécution des ordres de celui-ci. Ce commissaire fera office de président des Etats provinciaux.

Ces dispositions de la constitution de 1814 ont été reprises dans les grandes lignes par la constitution du royaume agrandi des Pays-Bas, créé en 1815 par le 
Congres de Vienne et résultant de la réunion des anciennes dix-sept provinces des Pays-Bas de l'époque de Charles-Quint. Cette réunion des Pays-Bas et de la Belgique n'a eu qu'une durée éphémère. Les membres belges de la commission chargée de la rédaction de la nouvelle constitution étaient surtout gênés par ces commissaires du roi qui devaient alors prendre le nom de gouverneurs. Ils en sont restés à une délimitation bien moins centraliste de leur rôle.

On ne pourrait dire que tout cela a donné à la province de grandes possibilités d'épanouissement. Tel n'était d'ailleurs pas le but poursuivi. La crainte que les provinces ne cherchent à restaurer leur splendeur ancienne a oeuvré dans le même sens. Ce qui, àmon sens, a été plus important, c'est que dans le nouveau système politique il n'y avait guère besoin d'un rôle important des provinces, étant donné l'ensemble des tâches réparties entre les divers échelons administratifs. Les provinces accomplissaient, comme ce fut longtemps le cas, beaucoup de choses dans le domaine de l'équipement hydraulique, l'une ou l'autre chose dans celui de l' amélioration des routes et elles s'occupaient de temps en temps d'une question reprise ultérieurement de façon plus approfondie par 1'Etat. Ici, il s'agissait d'un aspect de l'enseignement primaire, là, d'une question relative aux poids et mesures, parfois d'un problème lié à l'assistance aux malades mentaux. Mais les activités des provinces restèrent en général limitées.

Telle était la situation avant et longtemps encore après 1'année 1850, année tres importante pour les provinces. C'est en 1850 que fut promulguée une loi qui, sous le nom de Loi Provinciale (Provinciale Wet), a fixé le régime des provinces pendant plus d'un siècle. Cette loi avait été rendue nécessaire par la révision de la constitution de 1848. Elle définissait en termes clairs les lignes de force suivantes:

1. Les Etats provinciaux sont constitués par des élections générales.

2. Les assemblees des Etats sont publiques.

3. Les provinces obtiennent un budget propre et des ressources fiscales autonomes et limitées.

La Loi Provinciale de 1850 est 1'oeuvre du grand homme politique libéral Thorbecke à qui l'on doit aussi la Loi Communale. La codification de ces questions dans une loi mit fin aux trop grandes différences existant entre les diverses provinces. L'une des dispositions les plus marquantes est la réglementation selon laquelle les Etats provinciaux ne peuvent se réunir que deux fois par an (ce qui accroît l'importance de la tâche des Etats députés). Cette disposition est restée en vigueur pendant plus d'un siècle et elle a sans nul doute entravé le développement des provinces. Cette prescription a été abrogée en 1962. Cette année là, une nouvelle Loi sur les Provinces (Provinciewet) est entrée en application et est toujours en vigueur actuellement. Cette loi a accordé davantage de liberté d'action aux provinces. Les changements qui ont eu lieu sont cependant beaucoup plus étroitement liés à l'évolution sociale de 1'après-guerre dans son ensemble. 
La structure des provinces est relativement simple. Les Etats provinciaux sont élus une fois tous les quatre ans. Ils sont indissolubles. Ils constituent l'organe administratif général de la province. Le nombre de membres des Etats varie suivant 1'importance de la population de la province. Les Etats de la province la moins peuplée comptent 47 membres et ceux de la plus peuplée 83. La plupart des Etats se réunissent une fois par mois pendant un ou plusieurs jours. La commission permanente de la province est constituée par les Etats députés, composés de six à huit membres élus par les Etats provinciaux en leur sein pour la durée de la session. Les Etats provinciaux ont le droit de démettre de ses fonctions un membre des Etats députés, mais seulement quand il refuse de donner les renseignements nécessaires.

Le commissaire de la reine préside les Etats provinciaux; il a voix consultative dans cette assemblée. Il préside également les réunions des Etats députés. Dans cette dernière assemblée, il possède une voix, et même une voix décisive lorsque des affaires sont en cause et que les voies restent partagées au deuxième tour de scrutin.

Le commissaire a une double fonction. Cela n'est pas dû au fait qu'il est nommé par la couronne car, aux Pays-Bas, les bourgmestres le sont aussi. Il a une double fonction du fait qu'il est à la fois un organe de la province et un fonctionnaire de 1'Etat. Dans cette dernière fonction, il reçoit un mandat émanant du gouvernement central. Ce mandat lui donne pour mission de coordonner les secours en cas de catastrophe, le charge de faire des recommandations pour la nomination des bourgmestres et règle encore quelques autres questions mineures. En réalité, le commissaire est devenu au cours des années un organe avant tout provincial.

Des objections ont été formulées contre cette double fonction du commissaire; elles ne visent pas tellement la trop grande influence que peut avoir le gouvernement dans les affaires de la province, car elle n'y est pas liée, mais plutôt un prétendu manque de clarté en matière de responsabilité. La Deuxième Chambre a récemment adopté une proposition visant à modifier la constitution dans le sens suivant: la loi peut établir que le commissaire reçoit un mandat de 1'Etat.

Le problème de la centralisation et de la décentralisation doit être abordé du point de vue suivant: dans quelle mesure veut-on reconnaître des compétences déterminées aux autorités publiques centrales ou subordonnées.

Au cours des premières décennies de ce siècle, les Pays-Bas ont vécu dans une philosophie liée aux conceptions économiques libérales. Selon cette philosophie, 1'Etat s'abstenait le plus possible d'intervenir et de réglementer. La province et les communes vivaient aussi dans la même optique. Il y avait bien partout toutes sortes de questions étroitement liées à la vie quotidienne du citoyen et, lorsqu'il y avait quelque chose a régler, la commune était la première instance à intervenir. Je songe à l'adduction d'eau: les châteaux d'eau en témoignent dans un grand 
nombre de communes. De très nombreuses communes plus importantes se chargèrent de la fourniture de l'énergie électrique. Seules les communes les plus importantes abordèrent le raccordement au téléphone. Plus tard, on voit également la province assurer la fourniture de l'électricité et de l'eau aux petites communes mais, en général, pour toutes sortes d'équipements (salles de lecture et surtout écoles), l'initiative est laissée aux communes.

La crise économique des années 30 n'a pas immédiatement transformé les conceptions théoriques; mais la pratique a toutefois subi d'importantes modifications. L'Etat devait régler de nombreuses questions d'ordre économique. A la question de connaître la répartition de ce pouvoir, la réponse est aisée à donner. Le gouvernement central de La Haye s'appropria ce pouvoir sans que des objections ne soient soulevées. Les circonstances particulières de la période de la guerre peuvent, à mon avis, se passer de commentaire. Ces années ont profondément agité le pays ainsi que les provinces et les communes. Toutes sortes de mesures étrangères à 1'administration furent introduites. Elles y étaient trop étrangères pour retenir 1'attention ici.

$\mathrm{Au}$ lendemain de la guerre, le rétablissement de la vie sociale requiert toute l'attention. Toutes sortes de transformations sociales s'annoncent. Entretemps, la prospérité économique avait disparu; il faut tout reconstruire en partant de zéro. Il ne faut plus compter sur le commerce avec les territoires d'outremer. Il ne reste presque plus rien de la marine, etc. etc. La construction de logements a pris un retard effrayant; la crise du logement a atteint des proportions inimaginables. Ce n'est pas seulement l'urgence de la situation qui avait fait évoluer les idees dans le sens d'un renforcement de l'intervention des pouvoirs publics. Il allait de soi qu'il devait s'agir en l'occurrence des pouvoirs publics centraux; d'autre part, ce sont surtout les communes qui étaient impliquées lors de 1'application des lois. Les provinces l'étaient dans une moindre mesure.

Cette différence était logique. En premier lieu, c'est la commune qui est la plus proche de la population et, dans le domaine des services, elle est donc la première concernée. En deuxième lieu, les communes se sont entièrement ouvertes à cette nouvelle évolution. En troisième lieu, les communes se sont associées au sein d'une organisation collective, 1'Union des Communes Néerlandaises (Vereniging van Nederlandse Gemeenten). A ma connaissance, les 850 communes environ sont toutes affiliées à cette union. D'une façon digne d'admiration, cette union est devenue un très puissant bastion qui, par ses conseils aux communes et ses interventions énergiques au dehors, a accompli et continue d'accomplir un travail plus que méritoire pour les communes et leurs habitants.

Cette image continue de dominer même lorsque la situation a cessé d'être urgente. Le modèle suivi est demeuré la ligne directrice quand la prospérité s'est développée et que des problèmes bien différents des aménagements de secours se sont posés. 


\section{P.J. VERDAM}

J'esquisse ici au passage la situation aux environs de 1'année 1965: une tendance fortement centraliste lors de la prise de nombreuses mesures, dans laquelle la position de 'La Haye' domine tout. Les services de 1'Etat décentralisé sont de plus en plus nombreux; il s'agit de filiales ou d'inspections de services ministériels installés en province pour un ressort territorial déterminé, coïncidant souvent, mais pas toujours, avec les limites provinciales. Dans l'exécution des lois, les communes sont également impliquées et, dans une moindre mesure, les provinces.

Plus que de cette application des lois (appelée 'co-administration' dans ce pays), les communes et les provinces s'enorgeuillissent d'un certain degré d'autonomie dont elles disposent traditionnellement et qui consiste dans le pouvoir de régler leurs propres affaires. Mais il est difficile de définir le contenu de ces 'propres affaires'. En principe cela comprend tout, mais il existe toutefois une règle qui apporte une très importante restriction.

J'entends par là la règle selon laquelle une réglementation émanant de la commune est abolie par une réglementation émanant d'une autorité supérieure, province ou Etat, traitant de la même question. De la même façon, une ordonnance provinciale cède le pas à une loi. Un pouvoir supérieur peut donc écarter une réglementation émanant d'une instance inférieure, mais il faut tout de même posséder suffisamment d'autorité et de pouvoir réels pour cela. L'Etat y parvient sans difficulté. En revanche, une province y regarde à plusieurs reprises avant de supplanter un règlement communal. Les provinces étaient d'ailleurs assez mal placées pour agir de la sorte: jusqu'en 1962, les Etats ne se réunissaient pas plus de deux fois par an.

Puis les rouages tournent. A partir de 1965, on arrive peu à peu à la conclusion que quelques engrenages se bloquent. Les communes en avaient déjà pris conscience depuis longtemps; il arrivait fréquemment qu'une activité déterminée dépassait les capacités d'une seule commune. A plusieurs reprises, il était apparu nécessaire ou souhaitable que plusieurs communes entreprennent certaines choses ensemble. Une chose très utile en soi. Pour cette raison, dès 1950, une loi s'appliquant à ces affaires intercommunales avait vu le jour. Cette loi a institutionnalisé les formes de collaboration entre différentes communes. Selon cette loi, les communes peuvent créer une 'réglementation' intercommunale ${ }^{1}$ pour une tâche déterminée. Dans cette optique, une intercommunale consiste le plus souvent en une personne morale au sein de laquelle participent deux ou plusieurs communes dans le but de réaliser ensemble un projet déterminé.

Cette loi a très bien fonctionné mais son application était trop fréquente. Cela était une conséquence de la pléthore d'événements et de mesures qui nous ont assaillis. Les problèmes de plus en plus complexes de notre vie en société, la surpo-

1. 'Réglementation' intercommunale, après: intercommunale. 
pulation affectant principalement l'ouest de notre pays, ont été à 1'origine de la multiplication des lois et des ordonnances. Etant donné que, pour chaque question devant être traitée en commun, il fallait instaurer une intercommunale particulière, les dites intercommunales se multiplièrent à un rythme vertigineux. Les communes impliquées dans une cinquantaine de telles réglementations (et souvent avec des partenaires différents) ne sont pas l'exception. Cela ne pouvait continuer. A cela s'ajoute que la décentralisation figurait au programme de chaque gouvernement, bien qu'elle fut, dans la plupart des cas, bien vite rangée dans un placard bien gardé. Mais, ainsi, la situation se bloque. En 1971, le ministre Beernink a présenté un projet de loi destiné à rompre le cadre étroit de la loi sur les intercommunales. Elles ne devaient pas seulement convenir à une tache donnée, mais devaient aussi poursuivre un dessein plus général. Il apparut toutefois rapidement que cela ne suffisait pas par rapport à ce qui était recherché. Dans 1'intervalle, l'idée des 'régions' était née. Cette idée des régions fut favorisée par le fait que de telles intercommunales générales comprenaient toujours les mêmes communes. Cela conduisit à l'idée d'une division géographique du pays et de ses onze provinces en un nombre beaucoup plus élevé de régions. En 1974, le ministre De Gaay Fortman a exposé cette idée dans un schema structural comprenant 44 régions. Le projet était très attrayant. De cette façon, l' administration aurait été nourrie par la base. Les communes pourraient ainsi confier à ces régions des tâches plus nombreuses qui seraient donc des intercommunales transformées. Les onze provinces s'estompaient alors quelque peu à 1'horizon. Trois ou quatre grandes circonscriptions suffiraient à les remplacer. L'idée n'a pas tenu longtemps. La discussion soulevée à ce propos a mis en lumière les éléments suivants. Une solution de cet ordre ne déplaisait généralement pas aux communes tandis que les provinces, pressentant leur fin proche, adoptèrent un silence inquiet ou s'y opposèrent de façon radicale. Les deux conceptions étaient valables. Mais les objections furent décisives:

1. Les intercommunales sont difficiles à appliquer de façon satisfaisante sur le plan administratif. Pour leur administration, deux modèles sont à envisager. Le premier prévoit qu'elles soient administrées par un important conseil dont les membres seraient élus parmi les administrateurs des communes affiliées. Cela présente le désavantage de mettre la question hors de portée de la population, plus qu'il n'est souhaitable sur le plan démocratique, et de la rendre facilement opaque. Le second modèle consiste en un conseil élu de façon démocratique, mais alors la commune n'a plus aucune emprise sur son produit.

2. La deuxième difficulté était que les communes s'avéraient peu disposées à se décharger de tâches essentielies en faveur de ces régions.

3. La troisième objection soulevée était que la réalisation d'une telle carte de régions exigeait une modification de la constitution. Or, cela nécessite un délai de 


\section{P.J. VERDAM}

nombreuses années aux Pays-Bas car la dissolution de la Chambre est alors requise.

4. La quatrième objection était qu'il faudrait faire disparaître 1'appareil existant des onze provinces pour créer une nouvelle organisation beaucoup plus importante.

5. La cinquième objection était que le nombre de 44 régions était jugé beaucoup trop important. Cela nécessitait en outre la présence d'un certain nombre de circonscriptions nationales coiffant ces régions et alors apparaissait le spectre d'une administration à quatre niveaux.

Le ministre De Gaay Fortman modifia ses projets et s'engagea encore en 1975 dans une voie toute différente. Il réduisit considérablement le nombre des circonscriptions, ne parla plus de régions et proposa d'étendre le nombre des provinces de 11 à 24. Il écartait ainsi un grand nombre des objections soulevées contre les régions. Une révision de la constitution n'était plus nécessaire. Les appareils en place étaient utilisés moyennant quelques modifications. La transparence démocratique apparaissait mieux. Mais la critique restait dirigée sur le nombre. Ce nombre demeurait trop élevé pour des provinces placées immédiatement au-dessous du gouvernement central. De plus, si le ministre De Gaay Fortman avait bien dressé une liste de tâches qui ne seraient plus du ressort de la commune, la décentralisation des tâches centrales n'était pas réalisée en même temps et les promesses ne sont rien de concret.

Là-dessus, l'actuel ministre Wiegel est à nouveau intervenu en 1978 dans une note proposant de réduire encore plus le nombre des provinces et il fut alors question de 17 provinces. A ce stade de la discussion, on assiste à un déplacement des accents. Les nombres 44, 24 ou 17 sont fortement déterminés par des facteurs géographiques. Chacun veut consulter la carte, chacun examine la situation selon que sa localité est mise en question. Le concept de délégation de pouvoirs de 1'Etat aux provinces ou à des instances subordonnées a toujours donné lieu à de vifs échanges d'arguments, mais cela semblait toujours être de beaux discours.

Soudain, je n'en connais pas la raison précise, il souffle sur La Haye un vent de déconcentration. L'idée d'abandonner une grande quantité de travail d'exécution semble s'être imposée dans divers ministères. Tout devient tellement compliqué et tellement vétilleux. De sérieux efforts sont entrepris en vue d'élaborer des réglementations faisant plus largement appel aux provinces et aux communes. L'on confond parfois encore décentralisation et déconcentration, et l'on menace souvent de maintenir l'approbation ministérielle sur des points mineurs. Les communes doivent conserver le plus grand nombre possible de prérogatives. Des échelons intermédiaires artificiels n'apportent pas de solution. L'extension de l'étendue des communes est probablement nécessaire. La province doit éviter le plus possible de supplanter les communes; son action doit surtout se situer dans 
le domaine de la planification, de la coordination et de l'orientation et ne relève du domaine de l'exécution que lorsqu'il s'agit de tâches dépassant nettement l'envergure des communes.

Les Pays-Bas connaissent actuellement une administration assez centralisée. Une proposition plus précise du ministre Wiegel est attendue. Il réduira probablement encore le nombre proposé de provinces. Mais l'aspect de la carte cède le pas devant l'idée de la décentralisation. Si le ministre parvient à saisir à temps cette idéé renaissante et à lui donner forme, une réorganisation pourra revêtir une grande valeur pour l'administration de notre pays.

\section{Bibliographie}

S.J.R. de Monchy et A.H. Günther, Handboek voor het Nederlands provincierecht (2. éd., Zwolle, 1976).

C.W. van der Pot et A.M. Donner, Handboek voor het Nederlandse staatsrecht (10. éd., Zwolle, 1977). 


\title{
The Dutch Republic and Antiquity*
}

\author{
W. DENBOER
}

When Huizinga described the constitutional law of the Republic as 'a peculiar structure', he added that it was perhaps none the worse for the lack of a theoretical basis.1The same conclusion was reached by Fockema Andreae on the basis of solid arguments, and I shall not contest it.

The Union was 'in essence a defensive alliance of the northern provinces', ${ }^{2}$ but with the emphasis on alliance. And for this collaboration, examples were sought. Here Rome no longer sufficed and was even completely inadequate, because that city kept control wherever she encouraged alliances throughout her long history. But there were other examples: the Greek cities in the Peloponnesian League grouped around Sparta, the two Athenian Confederacies, the Boeotian League, and above all the Achaean and Aetolian Leagues formed under the threat of Macedonia, which were often at odds with each other, but (because he was one of the leaders of the former) were described by the great historian Polybius as political instruments. Machiavelli respected Polybius as a political thinker, and the rediscovery of his histories marks an important event in the intellectual history of

\footnotetext{
* The literature on the Union is vast and subject to periodic expansion (1879, 1929, and this year: De Unie van Utrecht. Wording en werking van een verbond en een verbondsacte, edited by S. Groenveld and H.L.Ph. Leeuwenberg (The Hague, 1979). For my limited purpose I have benefitted most from discussions with colleagues, among whom I wish to thank in particular Mrs. N. van Santen-Mout, S. Groenveld, I. Schöffer, and J. Woltjer for their patience in listening to my questions and for their instructive and clarifying comments. I was able to read J. Woltjer's 'De wisselende gestalten van de Unie' in manuscript, for which I am also indebted to the author. Finally, last but not least, I wish to express my gratitude to the Leids Universiteitsfonds for making it possible for me to have the help of a graduate assistant for six months. The way in which Eric Daams fulfilled this function was beyond praise, but nevertheless must not go unacknowledged here.
}

1. J. Huizinga, Nederlands beschaving in de zeventiende eeuw (Haarlem, 1941)46, 'Misschien was het er niet minder om, dat het van geen enkele staatstheorie was uitgegaan'.

2. S. J. Fockema Andreae, De Nederlandse staat onder de Republiek, Verhandelingen der Koninklijke Nederlandse Akademie van Wetenschappen, afdeling Letterkunde, Nieuwe Reeks, LXVIII, iii (2nd. ed., Amsterdam, 1969) 3. 